\title{
MEMORY, MARKET STABILITY AND THE NONLINEAR COBWEB THEOREM
}

\author{
J. M. GAFFNEY ${ }^{1}$ and C. E. M. PEARCE ${ }^{1}$
}

(Received 8 March, 2002)

\begin{abstract}
Carlson has shown that if the predicted price in the linear cobweb model is taken as the average of all previous actual prices, then stability results independently of parameter values provided only that the demand-curve gradient is less than that of the supply curve. This result has subsequently been generalised by Manning and by Holmes and Manning. We investigate the robustness of their results.
\end{abstract}

\section{Introduction}

It is now over half a century since the publication of Ezekiel's seminal article [5] on the cobweb model and a rich literature has explored some of its ramifications. Ezekiel drew attention to the fact that whether stability or instability arises in the naïve predictive model is dependent on the values of its parameters. The sequence of successive predicted prices in a cobweb model can be regarded as values in an iterated-function system, which is a type of dynamical system. It is therefore not entirely surprising that Jensen and Urban [9] were able to find a broad spectrum of complex behaviour for cobweb models, including stable, periodic orbits of arbitrary order and chaos. See also Artstein [1]. Chiarella [4] has used a nonlinear supply curve with exactly one inflection point to make plausible that chaotic behaviour may result in such models. Finkenstädt and Kuhbier [6] have considered the question of chaotic dynamics with respect to actual agricultural markets. This is the context in which the cobweb model can perhaps be expected to be at its best as a descriptor of actual behaviour, since as noted by Bull [2] there are often substantial, irreversible commitments to future sales, inventories which are difficult or costly to hold and the product is sold on an auction market.

\footnotetext{
'School of Applied Mathematics, The University of Adelaide, SA 5005, Australia; e-mail: jgaffney@maths.adelaide.edu.au and cpearce@maths.adelaide.edu.au. (C) Australian Mathematical Society 2004, Serial-fee code 1446-181 1/04
} 
As remarked by Holmes and Manning [8], economic systems include an additional feature not expected in physical and biological systems, namely, they involve rational or optimizing individuals who learn from their experience. Nerlove [12] allowed for memory in the linear cobweb model. Let $\hat{p}_{n}$ denote the predicted and $p_{n}$ the actual price in time period $n$. Then his adaptive-expectation assumption can be written

$$
\hat{p}_{n+1}=(1-\beta) \hat{p}_{n}+\beta p_{n} \quad(0<\beta \leq 1)
$$

and can be interpreted as saying that producers revise their previous expectation of price in each period in proportion to the difference between actual price and the previous expectation. This reduces to the naïve model in the case $\beta=1$. Nerlove found that this model extended the domain of parameter values for which stability arises. A discussion of rational learning with memory has been given by Muth [11]. See Friedman [7] for a further discussion.

In 1968 Carlson [3] made the dramatic discovery that if the predicted price in the original linear cobweb model is taken as the average of all previous actual prices, then stability results regardless of parameter values provided only that the demand-curve gradient be less than that of the supply curve. This result has been generalised by Manning [10] to the case of continuous and strictly monotone supply and demand curves intersecting at an equilibrium price $p^{e}$. In the case of primary economic interest, that of an increasing supply curve and a decreasing demand curve, a further assumption is involved, namely, that there is a maximum price $M$, at which demand is zero. Holmes and Manning [8] considered the replacement of this assumption with a boundedness condition involving the gradients of the supply and demand functions for the case of differentiable curves and have examined the behaviour of trajectories in the approach to equilibrium.

It is natural to put the question of the robustness of the basic Carlson-Manning result. In the case of a linear cobweb, Carlson investigated what happens when a uniform weighting over past actual prices is replaced by a geometric weighting ascribing less emphasis on recent prices, that is,

$$
\hat{p}_{n+1}=\sum_{t=0}^{n} r^{t} p_{t} / \sum_{t=0}^{n} r^{t} \quad(0<r<1) .
$$

He found that, apart from the exceptional case in which the market hits equilibrium in a finite number of steps, the market will always converge but not to the equilibrium price $p^{e}$. He stated that the result arises because the particular expectations hypothesis gives insufficient weight to recent errors in forecasting. This result contrasts with what happens with Nerlove's adaptive-expectation assumption, which can be expressed as

$$
\hat{p}_{n+1}=\beta \sum_{t=0}^{n-1} p_{n-t}(1-\beta)^{t}+(1-\beta)^{n} \hat{p}_{1}
$$


and which gives heaviest weighting to the most recent prices. As we noted, stability arises with this model only under additional assumptions on the parameter values. At first sight, the stability of the Carlson-Manning result thus appears delicately poised between instabilities associated with too much or too little weighting of recent prices.

For comparison with (1.1), the Carlson predictive model with uniform weighting can be written as

$$
\hat{p}_{n+1}=\frac{1}{n} p_{n}+\left(1-\frac{1}{n}\right) \hat{p}_{n}
$$

In this article we investigate the question of when stability occurs invariably for all parameter values under a general weighting schema

$$
\hat{p}_{n+1}=\beta_{n} p_{n}+\left(1-\beta_{n}\right) \hat{p}_{n} \quad\left(0<\beta_{n}<1\right) .
$$

With the empty product interpreted as unity, this may be expressed alternatively as

$$
\hat{p}_{n+1}=\sum_{t=0}^{n-1} \beta_{n-t} p_{n-t} \prod_{m=0}^{t-1}\left(1-\beta_{n-m}\right)+\hat{p}_{1} \prod_{m=1}^{n}\left(1-\beta_{m}\right),
$$

that is, a general moving-average form. It turns out that invariable stability is not an isolated anomaly associated with uniform weighting of past prices but arises for a broad spectrum of models of the form (1.2). Roughly speaking, we shall find that the key factors for stability are $\beta_{n} \rightarrow 0$ and $\sum \beta_{n}=\infty$, which are two of the most salient properties of $\beta_{n}=1 / n$. This is somewhat reassuring, as in practice a precisely even weighting would be impossible to achieve, if only because of rounding errors and the fact that the currency is a discrete rather than a continuous entity. Such fluctations, with $\beta_{n}$ regarded as a perturbation of $1 / n$, in any given trajectory of a market will not disturb invariable stability provided that they are not large enough to affect the two properties noted above. A trajectory may be regarded as a sample path of a stochastic process and the perturbations as stochastic noise. Although couched in an entirely deterministic framework, our analysis may thus be regarded as a stochastic analysis taken at sample-path level.

Although we are interested primarily in when convergence of $\left(p_{n}\right)$ and $\left(\hat{p}_{n}\right)$ to $p^{e}$ does and does not occur, our discussion uncovers a certain amount of additional information on the behaviour of these sequences. Basic ingredients in our argument are the equivalences $\hat{p}_{n}>p^{e}$ if and only if $p_{n}<p^{e}$ and $\hat{p}_{n}<p^{e}$ if and only if $p_{n}>p^{e}$, which are well known in the literature and are direct consequences of the monotonicity of the supply and demand curves.

First we establish the following simple result. 
THEOREM 1.1. Suppose the supply curve is strictly increasing and the demand curve strictly decreasing, that they are continuous, meeting at a price $p^{e}$, and that demand is zero at some finite price $M$. Suppose further that the predicted price is determined by (1.2). Then a sufficient condition for $p_{n} \rightarrow p^{e}$ and $\hat{p}_{n} \rightarrow p^{e}$ for all parameter values is that $\beta_{n} \rightarrow 0$ and $\sum \beta_{n}=\infty$.

The proof devolves upon the three possible cases listed below.

(a) $\hat{p}_{n}=p^{e}$ for some finite value $m$ of $n$. In this case $p_{n}=p^{e}$ and $\hat{p}_{n}=p^{e}$ for all $n \geq \boldsymbol{m}$ and there is nothing to prove.

(b) There are arbitrarily large values of $n$ with $\hat{p}_{n}, \hat{p}_{n+1}$ separated by $p^{e}$.

(c) $\hat{p}_{n}$ is on the same side of $p^{e}$ for all sufficiently large values of $n$.

In the following section we establish case (b) of the theorem. Section 3 provides a canonical representation which is convenient for analysis and which will also be used elsewhere. This leads to establishing part (c) of Theorem 1.1 in Section 4. In Section 5 we generalise Theorem 1.1 to Theorem 5.1 and make some further deductions. We conclude in Section 6 with a partial converse to Theorem 5.1.

\section{Proof of Theorem 1.1 in case (b)}

Proof. Since $\beta_{n} \rightarrow 0$, then for any $\epsilon>0$ we may choose $N$ sufficiently large that $n \geq N$ entails $\beta_{n}<\epsilon$ and $\beta_{n} M<p^{e} \epsilon$. Fix $\epsilon$ and choose $N$ correspondingly.

Suppose $n \geq N$ and $\hat{p}_{n}>p^{e}$. Then $p_{n}<p^{e}<\hat{p}_{n}$ so from (1.2) used twice we have

$$
\hat{p}_{n+1}<\left(1-\beta_{n}\right) \hat{p}_{n}+\beta_{n} \hat{p}_{n}=\hat{p}_{n}
$$

and

$$
\hat{p}_{n+1}>\left(1-\beta_{n}\right) \hat{p}_{n}>(1-\epsilon) p^{e} .
$$

Similarly if $n \geq N$ and $\hat{p}_{n}<p^{e}$ then

$$
\hat{p}_{n+1}<\left(1-\beta_{n}\right) p^{e}+\epsilon M<(1+\epsilon) p^{e}
$$

and

$$
\hat{p}_{n+1}>\left(1-\beta_{n}\right) \hat{p}_{n}+\beta_{n} \hat{p}_{n}=\hat{p}_{n} .
$$

On taking these inequalities together, we have that

$$
\hat{p}_{n}>p^{e} \text { implies } \hat{p}_{n+1} \in\left((1-\epsilon) p^{e}, \hat{p}_{n}\right)
$$

and

$$
\hat{p}_{n}<p^{e} \quad \text { implies } \hat{p}_{n+1} \in\left(\hat{p}_{n},(1+\epsilon) p^{e}\right)
$$


As there are values of $n$ arbitrarily large for which $\hat{p}_{n}, \hat{p}_{n+1}$ are separated by $p^{e}$, there must as a consequence of (2.1) and (2.2) be an integer $m$ such that

$$
\hat{p}_{n} \in\left((1-\epsilon) p^{e},(1+\epsilon) p^{e}\right)
$$

for all $n \geq m$. Since $\epsilon>0$ is arbitrary, we therefore have $\hat{p}_{n} \rightarrow p^{e}$ and hence also $p_{n} \rightarrow p^{e}$ as desired.

\section{Canonical form}

For the remainder of the paper it is convenient to employ a canonical form of (1.2) not involving $p_{n}$ explicitly. Suppose the demand $D_{n}$ at time $n$ is given by $D_{n}=f\left(p_{n}\right)$ and the supply $S_{n}$ by $S_{n}=g\left(\hat{p}_{n}\right)$. By market clearing, $f\left(p_{n}\right)=g\left(\hat{p}_{n}\right)$. The function $h=f^{-1} \circ g$ is strictly decreasing as a consequence of the properties of $f$ and $g$, and $p_{n}$ can be expressed as a function of $\hat{p}_{n}$ by

$$
p_{n}=h\left(\hat{p}_{n}\right)
$$

The definition of $\hat{p}_{n+1}$ can be written

$$
\hat{p}_{n+1}=\left(1-\beta_{n}\right) \hat{p}_{n}+\beta_{n} h\left(\hat{p}_{n}\right)
$$

Set $q_{n} \equiv \hat{p}_{n}-p^{e}$, so

$$
q_{n+1}=\left(1-\beta_{n}\right) q_{n}+\beta_{n} \phi\left(q_{n}\right)
$$

where $\phi(q) \equiv h\left(q+p^{e}\right)-p^{e}$. The function $\phi$ inherits the strictly decreasing property of $h$. Further, since the supply and demand curves intersect at price $p^{e}$, we have $h\left(p^{e}\right)=p^{e}$ and so

$$
\phi(q) \begin{cases}<0 & \text { for } q>0 \\ =0 & \text { for } q=0 \\ >0 & \text { for } q<0\end{cases}
$$

We have from this result and (3.1) that

$$
q_{n+1} \begin{cases}<\left(1-\beta_{n}\right) q_{n} & \text { for } q_{n}>0 \\ >\left(1-\beta_{n}\right) q_{n} & \text { for } q_{n}<0 .\end{cases}
$$

This gives in particular that when $q_{n}$ and $q_{n+1}$ are of the same sign

$$
\left|q_{n+1}\right|<\left(1-\beta_{n}\right)\left|q_{n}\right|
$$




\section{Proof of Theorem 1.1 for case (c)}

PROOF. Since $q_{n}$ and $q_{n+1}$ have the same sign for all $n$ sufficiently large, for all $n \geq N$, say, (3.2) gives

$$
\left|q_{n+1}\right|<\left(1-\beta_{n}\right)\left|q_{n}\right| \text { for } n \geq N
$$

and so

$$
\left|q_{n+1}\right|<\left|q_{N}\right| \prod_{m=N}^{n}\left(1-\beta_{m}\right)
$$

for $n \geq N$. By a result of elementary analysis, the product on the right-hand side tends to zero with $n \rightarrow \infty$ if and only if $\sum \beta_{n}=\infty$. Hence $\sum \beta_{n}=\infty$ suffices to give $q_{n} \rightarrow 0$, that is, $\hat{p}_{n} \rightarrow p^{e}$ and hence also $p_{n} \rightarrow p^{e}$ as required.

In the proof for case (c) we found that for sufficiently large $n$ we have either $\hat{p}_{n}>\hat{p}_{n+1}>p^{e}$ or $\hat{p}_{n}<\hat{p}_{n+1}<p^{e}$. In either event $\left(\hat{p}_{n}\right)$ is therefore an eventually monotone and bounded sequence and so is convergent. However it is not immediate that the limit must be $p^{e}$. The thrust of our proof is establishing that the conditions on $\left(\beta_{n}\right)$ require the limit to actually be $p^{e}$. This point is not addressed by Manning [10] and to that extent his proof is incomplete. Theorem 1.1 subsumes his situation with $\beta_{n}=1 / n$, since $1 / n \rightarrow 0$ and $\sum 1 / n=\infty$.

\section{Further generalisations}

The assumption concerning the existence of an upper limiting price $M$ can be replaced by one imposing suitable restrictions on the growth of $\phi$. We do not need a condition as strong as the differentiability of $\phi$. An appropriate condition is that for all $q$ for which $\phi$ is defined, $\phi$ satisfies a Lipschitz condition

$$
|\phi(q)|<C|q|, \quad \text { for } C \text { some finite constant. }
$$

This is a global condition on growth. When $\phi$ is differentiable it may even possess gradient $-\infty$ at some isolated points (other than zero) without this extreme local behaviour violating the global constraint.

THEOREM 5.1. Suppose the conditions of Theorem 1.1 hold except that the requirement that demand vanishes at some finite price $M$ is replaced by the assumption that $\phi$ satisfies a Lipschitz condition (5.1) for all sufficiently large $n$. Then the conclusions of Theorem 1.1 still apply. Further, the convergence is eventually monotone. 
PROOF. The existence of $M$ is invoked in the proof of Theorem 1.1 only in case (b). Now under (5.1), $q_{n}>0$ implies by (3.1) that

$$
q_{n+1}>\left[1-\beta_{n}(1+C)\right] q_{n}>0 \text { for all } n \text { sufficiently large, }
$$

and similarly $q_{n}<0$ implies that

$$
q_{n+1}<\left[1-\beta_{n}(1+C)\right] q_{n}<0 \text { for all } n \text { sufficiently large. }
$$

Thus $\left(q_{n}\right)$ is eventually of fixed sign and then $\left|q_{n+1}\right|<\left|q_{n}\right|$. The former conclusion implies that case (b) does not arise, giving the desired result.

As noted, while the condition $\beta_{n} \rightarrow 0$ is used to show that case (b) does not arise, this condition is not used further in the treatment of case (c). In the context of either Theorem 1.1 or Theorem 5.1, suppose we know that $\beta_{n} \nrightarrow 0$ but case (b) does not arise. Then since $\beta_{n} \nrightarrow 0$ implies $\sum \beta_{n}=\infty$, we have automatically that $\left(\hat{p}_{n}\right)$ and $\left(p_{n}\right)$ must converge to $p^{e}$ for all parameter values. Such possibilities are of interest. Holmes and Manning [8] comment, with examples, on the extreme slowness with which convergence to $p^{e}$ may occur. The reason for this, at least in case (c), is apparent from (4.1). For $\beta_{n}=1 / n,(4.1)$ gives

$$
\left|q_{n+1}\right|<\left|q_{N}\right| N / n
$$

so that $\left|\hat{p}_{n}-p^{e}\right| \rightarrow 0$ like $1 / n$, which is very slow. If $\beta_{n}$ were bounded away from zero, then the convergence would be exponentially fast.

The Nerlove adaptive-expectation assumption has $\beta_{n} \equiv \beta \nrightarrow 0$. If case (b) did not arise we would have convergence of $\left(p_{n}\right)$ to $p^{e}$ for all parameter values, which is known from the linear cobweb model not to be the case. Hence it is an easy corollary from that fact alone that in general case (b) must arise for some parameter values, that is, there can be continued oscillation about $p^{e}$ in the Nerlove model as $p_{n} \rightarrow p^{e}$.

In connection with rates of convergence to stability, we remark that in general (4.1) indicates that, at least when case (c) applies, convergence to $p^{e}$ may be hastened by replacing $\beta_{n}=1 / n$ by something that converges to zero more slowly, such as $\beta_{n}=n^{-\alpha}$ for $0<\alpha<1$.

\section{A converse result}

If $\sum \beta_{n}<\infty$ (or equivalently $\prod\left(1-\beta_{n}\right)>0$ ) the situation is more complicated and the behaviour of the sequence $\left(\hat{p}_{n}\right)$ can depend on the initial estimate $\hat{p}_{0}$, the precise functional forms of $f$ and $g$ and on the sequence $\left(\beta_{n}\right)$ used. The following is a partial converse to Theorem 1.1 . 
THEOREM 6.1. Suppose the supply and demand curves are as in Theorem 5.1, with $\phi$ satisfying a Lipschitz condition (5.1). Assume the predicted prices are governed by (1.2) and that $\hat{p}_{1} \neq p^{e}$. If

$$
(C+1) \beta_{n}<1 \text { for all } n \text { and } \sum \beta_{n}<\infty
$$

then it cannot happen that $\left(\hat{p}_{n}\right),\left(p_{n}\right)$ converge to $p^{e}$.

PROOF. Under the Lipschitz condition, we have with the argument of Theorem 5.1 that $\left|q_{n+1}\right|>\left|q_{n}\right|\left[1-(C+1) \beta_{n}\right]$ for all $n$, so that

$$
\left|q_{n+1}\right|>\left|q_{1}\right| \prod_{m=1}^{n}\left[1-(C+1) \beta_{m}\right] .
$$

Since $\sum \beta_{n}<\infty$ we have $\sum(C+1) \beta_{n}<\infty$, so that the product on the right-hand side converges to a nonzero limit as $n \rightarrow \infty$. As $\hat{p}_{1} \neq p^{e}$ we have $q_{1} \neq 0$. Hence $q_{n} \nrightarrow 0$ and consequently $\hat{p}_{n} \nrightarrow p^{e}$ and $p_{n} \nrightarrow p^{e}$ as required.

If (5.1) holds, then $\sum \beta_{n}<\infty$ entails that $(C+1) \beta_{n}<1$ for all $n$ sufficiently large. Suppose we replace $(6.1)$ by $\sum \beta_{n}<\infty$. The difference made to Theorem 6.1 by this weakening of (6.1) is the introduction of the possibility that the predicted price achieves the value $p^{e}$ in some finite number of steps.

Under the conditions of Theorem 6.1, we have with the argument of Theorem 5.1 that $\left(q_{n}\right)$ is of constant sign and by (3.2) that $\left|q_{n+1}\right|<\left|q_{n}\right|\left(1-\beta_{n}\right)$. The sequence $\left(\left|q_{n}\right|\right)$ is a monotone decreasing sequence and bounded below and so is convergent. As $q_{n} \nrightarrow 0$ and is of constant sign, we thus have that $\left(q_{n}\right)$ converges to a nonzero limit. Thus Theorem 6.1 describes a situation in which $\left(p_{n}\right)$ converges to a limit different from $p^{e}$. The possibility of this sort of phenomenon appears to have been unremarked in the literature except for a footnote in Carlson [3], for which, as observed earlier, $\beta_{n} \nrightarrow 0$. These considerations make it clear that one should not infer from the convergence of a predictive schema that the limiting price is $p^{e}$, at least, not without some attention being given to the general properties of the sequence of weights involved in the schema.

\section{References}

[1] Z. Artstein, "Irregular cobweb dynamics", Econ. Lett. 11 (1983) 15-17.

[2] C. Bull, "Expectations of others' expectations", in Individual forecasting and aggregate outcomes. 'Rational expectations' examined (eds. R. Frydman and E. S. Phelps), (Cambridge University Press, Cambridge, 1983) 66-68.

[3] J. A. Carlson, "An invariably stable cobweb model", Rev. Econ. Stud. 35 (1968) 360-362. 
[4] C. Chiarella, "The cobweb model. Its instability and the onset of chaos", Econ. Model. 5 (1988) 377-384.

[5] M. Ezekiel, "The cobweb theorem", Q. J. Econ. 52 (1938) 255-280.

[6] B. Finkenstädt and P. Kuhbier, "Chaotic dynamics in agricultural markets", in Nonlinear methods in economic dynamics and optimal control, International Conference on Operations Research, Vienna (1990) (eds. G. Feichtinger and R. F. Hartl), Ann. Oper. Res. 37 (1992) 73-96.

[7] B. M. Friedman, "Optimal expectations and the extreme information assumptions of 'rational expectations' macromodels", J. Monetary Econ. 5 (1979) $27-41$.

[8] J. M. Holmes and R. Manning, "Memory and market stability: the case of the cobweb", Econ. Lett. 28 (1988) 1-7.

[9] R. V. Jensen and R. Urban, "Chaotic price behaviour in a non-linear cobweb model", Econ. Lett. 15 (1984) 235-240.

[10] R. Manning, "A generalization of a cobweb theorem", Rev. Econ. Stud. 36 (1971) 123-125.

[11] W. Muth, "Rational expectations and the theory of price movements", Econometrica 29 (1961) 315-335.

[12] M. Nerlove, "Adaptive expectations and cobweb phenomena", Q. J. Econ. 22 (1958) 227-240. 